\title{
Antiphosphatidylethanolamine Antibodies and Deep Vein Thrombosis in Lupus Patients with Antiphospholipid Syndrome
}

\author{
Simona Caraiola ${ }^{1,2}$, Ciprian Jurcuț $^{3}$, Alina Dima ${ }^{2 *}$, Cristian Băicuș ${ }^{1,2}$, \\ Anda Băicuș ${ }^{1}$ \\ 1. Carol Davila University of Medicine and Pharmacy, Bucharest, Romania \\ 2. Colentina Research Center, Colentina Clinical Hospital, Bucharest, Romania \\ 3. Dr. Carol Davila Central University Emergency Military Hospital, Bucharest, Romania
}

\begin{abstract}
Backgroud. Which factors determine venous thrombotic events in some antiphospholipid syndrome (APS) patients and arterial thrombosis or conditions related to pregnancy in others has not been established yet. Purpose. The aim of this study was to search the antiphospholipid antibodies (APLAs) correlates in regard to deep vein thrombosis (DVT) in patients with systemic lupus erythematosus (SLE) and APS. Methods. Twenty-nine patients fulfilling the criteria of both SLE and APS were included. Complete anamnesis and clinical examination was performed on inclusion. Also, for all patients, disease activity was assessed by the SLEDAI score. An extended APLAs profile, ten Abs, was searched. Results. The titers of IgG anticardiolipin ( $a C L)$, IgG anti- $\beta 2$ glycoprotein I (aß2G$P I), \operatorname{Ig} G$ antiphosphatidylethanolamine (aPE), and also of $\operatorname{Ig} G$ antiprothrombin (aPT) were significant higher in patients with DVT history. After analysis by ROC curve and univariate logistic regression, the strongest association was found for IgG aPE. Also, in multivariate analysis, SLEDAI score correlated with the DVT antecedents. Conclusions. IgG aPE might be involved in DVT pathogenic pathways in patients with SLE and APS as their titers remain significantly higher in patients with previous DVT. Lupus patients with DVT events represent a subgroup of patients with more severe underlying pathology.
\end{abstract}

Keywords: antiphosphatidylethanolamine antibodies, antiphospholipid syndrome, deep vein thrombosis, systemic lupus erythematosus

Received: $16^{\text {th }}$ November 2017; Accepted: $14^{\text {th }}$ February 2018; Published: $7^{\text {th }}$ March 2018

\section{Background}

Most recent antiphospholipid syndrome (APS) diagnostic criteria consider two main characteristics. On the one hand, the clinical manifestations and, on the other hand, the persistent positivity for at least one of the follow- ing antiphospholipid antibodies (APLAs): lupus anticoagulant (LAC), anticardiolipin antibodies $(\mathrm{aCL})$, or anti- $\beta 2$ glycoprotein I antibodies (aß2GPI) [1]. Moreover, several studies regarding the diagnostic and prognostic impact of the so-called non-criteria APLAs were published

*Corresponding author: Alina Dima, Colentina Clinical Hospital, Bucharest, Romania

E-mail: alina_dima@outlook.com 
$[2,3]$, but their role in the APS pathogenesis remains unsettled [4].

Systemic lupus erythematosus (SLE) is the most frequent autoimmune disease associated with APS and also, the pathogenesis of these two clinical conditions seems to be intricate [5]. There are, however, few studies concerning the role of non-criteria APLAs in SLE patients $[6,7]$.

We analyzed here the relation between the history of deep vein thrombosis (DVT) in patients with APS associated with SLE with a large spectrum of antibodies (Abs), both criteria and non-criteria APLAs.

\section{Methods}

\section{Cohort study}

The study was conducted on patients fulfilling the 2012 SLICC SLE criteria [8] as well as the 2006 Sydney APS criteria [1]. Twenty-nine patients diagnosed with both APS and SLE, hospitalized in Colentina Clinical Hospital, Bucharest were consecutively enrolled. All patients signed an informed consent on inclusion. This study was approved by the Clinical Research Ethics Committee of the Carol Davila University of Medicine and Pharmacy Bucharest.

\section{Variables}

For all patients, the same clinical and biological data were registered in order not to alter the homogeneity of the group. Systemic Lupus Erythematosus Disease Activity Index (SLEDAI) [9] score was assessed on recruitment in all cases.

The criteria and non-criteria APLAs profile searched for were as follows: IgG and IgM serotypes of anticardiolipin antibodies $(\mathrm{aCL})$, anti- $\beta 2$ glycoprotein I antibodies (aß2GPI), antiphosphatidylserine antibodies (aPS), anti phosphatidylethanolamine antibodies (aPE), respectively antiprothrombin antibodies (aPT). All determinations were made by ELISA - Aesku Diagnostics, Wendelsheim, Germany using the analyzer
Chemwell 2910, Awareness Technology, Palm City, Florida, USA. A cut-off of $18 \mathrm{UI} / \mathrm{ml}$ was used in all tested for defining Abs positivity.

\section{Statistical analysis}

Categorical values are expressed as numbers and percentage and quantitative ones as mean \pm standard deviation (SD) or median (minimum; maximum), med (min; max) according to their distribution. In bivariate analysis, the chi-square test was used to test differences for categorical variables, T-independent test for the normally distributed, while Mann-Whitney for the non-normally distributed variables. A p-value less than 0.05 was considered significant. Odds ratios (ORs) and 95\% confidence intervals (CIs) were calculated when appropriate. Moreover, the area under curve (AUC) by ROC curve was used to evaluate the relation between APLAs titers and DVT presence. Multivariate analysis by logistic regression (stepwise-forward method, adjusted to the small sample) was tested. Also, univariate logistic regression with DVT as dependent variable and each APLAs as independent one was done. All analysis was performed by SPSS 16.0.

\section{Results}

\section{Cohort study}

Demographic, clinical, and laboratory characteristics are summarized in Table 1. A total of 29 patients were included in the study, with net feminine predominance (96.6\%). The mean age on inclusion was 45.2 years.

Venous thrombosis was present in the disease history of 10 patients (34.5\%); for the rest, other clinical events, respectively arterial thrombosis or gestation pathology, sustained the APS diagnosis. There was no acute DVT event during the enrollment period of the patients included. Figure 1 presents the number of patients with the most important overlapping APS diagnostic clinical events recorded, namely DVT, arterial thrombosis, and obstetrical events. In 11 patients 
Table 1. Descriptive data $(n=29)$

\begin{tabular}{lc}
\hline Characteristic & \\
\hline Gender, no F (\%) & $28(96.6)$ \\
\hline Age at inclusion, years mean \pm SD & $45.2 \pm 11.1$ \\
\hline Age at APS's diagnosis, years mean \pm SD & $34.3 \pm 10.7$ \\
\hline Disease duration, years med (min; max) & $8.0(1.0 ; 32.0)$ \\
\hline Deep vein thrombosis, no (\%) & $10(34.5)$ \\
\hline Recurrent venous thrombosis, no (\%) & $2(6.9)$ \\
\hline Arterial thrombosis, no (\%) & $14(48.3)$ \\
\hline Gestation pathology, no (\%) & $10(34.5)$ \\
\hline Other thrombotic events, no (\%) & $11(44.9)$ \\
\hline LAC, no (\%) & $28(96.6)$ \\
\hline aCL, no (\%) & $8(27.6)$ \\
\hline aß2GPI, no (\%) & $6(20.7)$ \\
\hline LAC + aCL, no (\%) & $7(24.1)$ \\
\hline LAC + a $\beta 2 G P I$, no (\%) & $6(20.7)$ \\
\hline aCL + a $32 \mathrm{GPI}$, no (\%) & $4(13.8)$ \\
\hline
\end{tabular}

F - feminine; no - number; APS - antiphospholipid syndrome; aCL - anticardiolipin; a $32 \mathrm{GPI}$ - anti- $\beta 2$ glycoprotein I; LAC - lupus anticoagulant; med - median; min - minim; max - maxim; SD - standard deviation.

other APS events were noted, e.g. superficial vein thrombosis, venous thrombosis in other places than lower limbs (retinal, cava vein). The med (min; max) time from the DVT events oc- currence to the inclusion in the study was $7(0.5$; 14) years.

LAC positivity, twice determinate, was the most frequent APS biological marker present in

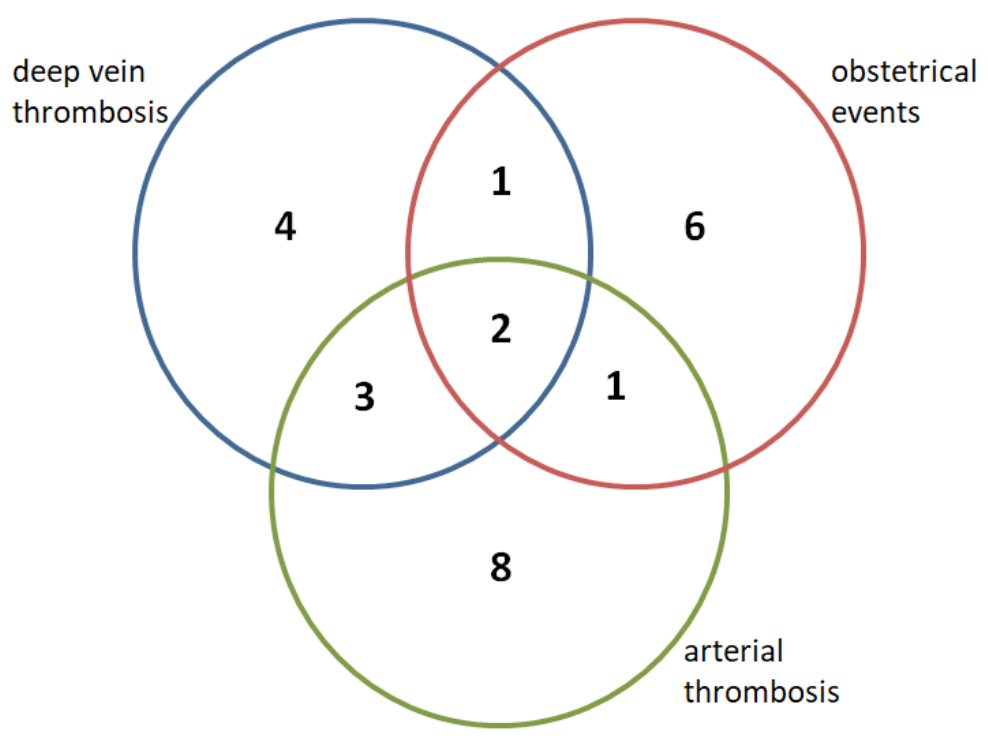

Figure 1. Number of patients included in regard to the clinical pathogenic events of antiphospholipid syndrome used for diagnosis (history of deep vein thrombosis, arterial thrombosis, and obstetrical events) ${ }^{*}$

* other thrombotic events were present in 11 patients (e.g. superficial vein thrombosis) 
patients' history (96.6\%). It was associated with the aCL positivity in 7 patients $(24.1 \%)$.

For further analysis, the studied group was divided into two subgroups: patients with history of DVT (10 patients) and patients without medical history of DVT (19 patients).

\section{DVT traditional risk factors}

In regard to the traditional risk factors of thrombosis, no significant differences were found between the two subgroups analyzed: gen$\operatorname{der}(\mathrm{F} / \mathrm{M}$ : $10 / 0$ vs. $18 / 1 ; \mathrm{p}>0.05)$; smoking (1/ 9 vs. $5 / 14 ; \mathrm{p}>0.05)$, body mass index $(24.5 \pm$ 5.8 vs. $\left.26.1 \pm 6.4 \mathrm{~kg} / \mathrm{m}^{2} ; \mathrm{p}>0.05\right)$; waist circumference $(98.9 \pm 19.0$ vs. $89.1 \pm 18.5 \mathrm{~cm})$ and waist/ hip ratio $(0.9 \pm 0.1$ vs. $0.8 \pm 0.1), \mathrm{p}>0.05$. (Table 2)

\section{Nonspecific inflammatory syndrome}

None of the parameters of nonspecific inflammatory syndrome, namely erythrocyte sedimentation rate (ESR), fibrinogen, and C-reactive protein (CRP) was found to be significantly dif- ferent in patients with history of DVT when compared to arterial thrombosis or obstetric events [with DVT/ without DVT: $19.0(3.0$ - 54.0) vs. $14.0(3.0-62.0) \mathrm{mm} / \mathrm{h}, 355.7 \pm 69.2$ vs. $318.2 \pm$ $73.3 \mathrm{mg} / \mathrm{dl}$, respectively $6.5(1.0-59.0)$ vs. 2.0 (0.0 - 15.0) mg/, $\mathrm{p}>0.05$ ] (see Table 2).

\section{Disease activity}

The baseline SLEDAI score results were significantly higher in the subgroup of patients with DVT history: $7.0(0.0-22.0)$, respectively 2.0 (0.0 - 12.0) points (Table 2$)$.

\section{ALPAs relation with DVT events}

From the extended profile of APLAs analyzed, a total of ten Abs, only the IgM aßGPI were significantly more frequently found in positive titer in patients with history of DVT: OR $(95 \% \mathrm{CI})$ $=12.0(1.1-129.4), \mathrm{p}=0.04$. No significant difference was observed when the positivity of any of the other APLAs tested was searched (data not show). Further, for four of the APLAs tested (IgG aCL, IgG aß2GPI, IgG aPE, and IgG aPT), we

Table 2. General characteristics and traditional risk factors for deep vein thrombosis in the two subgroups (with/ without deep vein thrombosis)

\begin{tabular}{lccc}
\hline Characteristic & $\begin{array}{c}\text { with DVT } \\
\mathbf{n}=\mathbf{1 0}\end{array}$ & $\begin{array}{c}\text { without DVT } \\
\mathbf{n}=\mathbf{1 9}\end{array}$ & p-value \\
\hline Age at inclusion, years mean \pm SD & $48.4 \pm 10.8$ & $43.6 \pm 11.2$ & $0.37^{\mathbb{P}}$ \\
\hline Age at APS's diagnosis, years mean \pm SD & $31.9 \pm 9.3$ & $35.5 \pm 11.4$ & $0.28^{\mathbb{P}}$ \\
\hline Disease duration, years med (min - max) & $15.0(1.0-33.0)$ & $6.0(1.0-29.0)$ & $0.01^{*}$ \\
\hline Gender, no F/ M & $10 / 0$ & $18 / 1$ & $0.04^{\varepsilon}$ \\
\hline Smoking, no Y/ N & $1 / 9$ & $5 / 14$ & $0.63^{\varepsilon}$ \\
\hline BMI, kg/m & $24.5 \pm 5.8$ & $26.1 \pm 6.4$ & $0.50^{\mathbb{P}}$ \\
\hline Waist circumference - feminine sex, cm & $98.9 \pm 19.0$ & $89.1 \pm 18.5$ & $0.20^{\mathbb{P}}$ \\
\hline Waist-hip ratio - feminine sex & $0.9 \pm 0.1$ & $0.8 \pm 0.1$ & $0.24^{\mathbb{P}}$ \\
\hline ESR, mm/ h med (min - max) & $19.0(3.0-54.0)$ & $14.0(3.0-62.0)$ & $0.27^{*}$ \\
\hline Fibrinogen, mg/ dl mean \pm SD & $355.7 \pm 69.2$ & $318.2 \pm 73.3$ & $0.19^{\mathbb{P}}$ \\
\hline CRP, mg/ l med (min - max) & $6.5(1.0-59.0)$ & $2.0(0.0-15.0)$ & $0.06^{*}$ \\
\hline SLEDAI, points med (min - max) & $7.0(0.0-22.0)$ & $2.0(0.0-12.0)$ & $0.01^{*}$ \\
\hline
\end{tabular}

p-value significant $<0.05$; APS - antiphospholipid syndrome; CRP - C-reactive protein; DVT - deep vein thrombosis; ESR - erythrocyte sedimentation rate; med - median; min - minim; max - maxim; SD - standard deviation; SLE - systemic lupus erythematosus; SLEDAI - systemic lupus erythematosus disease activity index.

PT-independent test ${ }^{*}$ Mann-Whitney test ${ }^{\circledR}$ Chi-square test 
found significantly higher titers, when analyzed in absolute number, in the serum of patients with history of DVT (data presented in Table 3).

In multivariate analysis, after introducing in turn each of the APLAs studied (positive/ negative), SLEDAI score, and also the inflammatory syndrome parameters, only the SLEDAI score remained significantly associated with the presence of DVT history $(\mathrm{p}<0.05)$. When analyzing by the ROC curve, we found the best association for the IgG aPE titer, 0.879 (0.755-1.003). Also, in univariate logistic regression the IgG aPE presented the best association with DVT history, $2.383(1.198-4.741)$, as presented in Table 4.

Table 3. Titers of the antiphospholipid antibodies in the two subgroups (with/ without deep vein thrombosis)

\begin{tabular}{lccc}
\hline Characteristic & $\begin{array}{c}\text { with DVT } \mathbf{n}=\mathbf{1 0} \\
\text { med }(\mathbf{m i n}-\mathbf{m a x})\end{array}$ & $\begin{array}{c}\text { without DVT n=19 } \\
\text { med (min - max) }\end{array}$ & p-value* \\
\hline IgG aCL & $3.0(0.0-64.0)$ & $1.0(0.0-20.0)$ & 0.01 \\
\hline IgM aCL & $9.5(0.0-41.0)$ & $3.0(0.0-26.0)$ & 0.11 \\
\hline IgG aß2GPI & $5.5(1.0-79.0)$ & $3.0(0.0-12.0)$ & 0.05 \\
\hline IgM aß2GPI & $9.0(2.0-300.0)$ & $6.0(0.0-36.0)$ & 0.11 \\
\hline IgG aPS & $2.5(1.0-112.0)$ & $2.0(1.0-40.0)$ & 0.14 \\
\hline IgM aPS & $7.5(0.0-31.0)$ & $3.0(1.0-26.0)$ & 0.09 \\
\hline IgG aPE & $5.0(3.0-151.0)$ & $2.0(1.0-7.0)$ & $<0.001$ \\
\hline IgM aPE & $11.5(3.0-202.0)$ & $6.0(0.0-58.0)$ & 0.07 \\
\hline IgG aPT & $5.0(2.0-20.0)$ & $3.0(1.0-7.0)$ & 0.05 \\
\hline IgM aPT & $5.5(0.0-10.0)$ & $3.0(0.0-13.0)$ & 0.18 \\
\hline
\end{tabular}

p-value significant $<0.05$; aCL - anticardiolipin; a $2 \mathrm{GPI}-$ anti- $\beta 2$ glycoprotein I; aPE - antiphosphatidylethanolamine; aPS - antiphosphatidylserine; aPT - antiprothrombin; DVT - deep vein thrombosis.

*Mann-Whitney test

Table 4. Antiphospholipid antibodies titers as predictors for deep vein thrombosis events (analysis by the ROC curve)

CI 95\%

CI 95\%

\begin{tabular}{lcccccc} 
Characteristic & AUC & Inf & Sup & OR & inf & sup \\
\hline IgG aCL & 0.771 & 0.583 & 0.959 & 1.095 & 0.960 & 1.248 \\
\hline IgM aCL & 0.682 & 0.462 & 0.902 & 1.084 & 0.986 & 1.192 \\
\hline IgG a 2 GPI & 0.716 & 0.511 & 0.921 & 1.241 & 0.943 & 1.634 \\
\hline IgM aß2GPI & 0.684 & 0.473 & 0.896 & 1.029 & 0.956 & 1.107 \\
\hline IgG aPS & 0.663 & 0.453 & 0.874 & 1.040 & 0.974 & 1.110 \\
\hline IgM aPS & 0.695 & 0.475 & 0.914 & 1.098 & 0.987 & 1.222 \\
\hline IgG aPE & 0.879 & 0.755 & 1.003 & 2.383 & 1.198 & 4.741 \\
\hline IgM aPE & 0.711 & 0.512 & 0.909 & 1.032 & 0.991 & 1.074 \\
\hline IgG aPT & 0.718 & 0.499 & 0.938 & 1.448 & 0.957 & 2.192 \\
\hline IgM aPT & 0.653 & 0.440 & 0.865 & 1.134 & 0.908 & 1.418 \\
\hline
\end{tabular}

aCL - anticardiolipin; AUC - aria under curve; a 32 GPI - anti- $\beta 2$ glycoprotein I; aPE - antiphosphatidylethanolamine; aPS - antiphosphatidylserine; aPT - antiprothrombin; CI - confidence interval; DVT - deep vein thrombosis; inf - inferior; sup - superior. 


\section{Discussions}

APLAs are some of the most encountered Abs in SLE patients, and their presence is related to thrombosis and recurrence of thrombosis [10]. Of these, LAC seems to carry the greater risk for either arterial or venous thrombotic event [11]. Baseline LAC positivity appears to bring up a 42 $\%$ associated risk of DVT over a 20 -year period [12]. Moreover, aß2GPI was demonstrated to be associated with DVT in patients with APS associated with SLE [13]. The presence of IgG aCL in medium and high titer might also increases the risk of thrombosis [14]. Some of the non-criteria Abs, such as aPT may develop LAC activity and their presence might be related to APS clinical events [15]. Regarding the aPE significance in APS, we do not have enough data yet. In some research, their presence is not associated with clinical APS events [16]. There is also evidence of aPE association with venous thrombotic events [17]. aPE has also been described in a subgroup of patients with clinical APS features, but without serological positive criteria APLAs; patients classified as "seronegative" APS. In these cases, non-criteria APLAs, including aPE, could be identified [18]. The results of studies regarding APLAs expression and significance in APS are divergent and one reason might be the lack of standardization of the laboratory determinations; so, efforts have been made in recent years to reach accuracy in the APS diagnosis [19].

In our analysis, we found significantly higher titers in the serums of patients with previous DVT for the criteria APLAs, IgG aCL and IgG aß2GPI, but also for the non-criteria APLAs, IgG aPE and IgG aPT. After analysis by ROC curve, the IgG aPE showed the strongest association with the history of DVT.

Both disease activity and inflammatory syndrome are characterized by high variability over time in SLE patients. Moreover, DVT events are accompanied by inflammatory reactions. In our study, we did not identify higher levels of the inflammation parameters in patients with DVT history. On the contrary, patients with previous DVT presented higher disease activity on inclusion, as expressed by the SLEDAI score results. We can only emphasize that lupus patients with APS clinically expressed as DVT might represent a subgroup of patients with more severe underlying pathology.

In the present research a large number of criteria and non-criteria APLAs were tested in SLE patients with APS from a single center. There are, however, several limitations to this study. The most important is the low number of patients included, which did not allow the assessment of any regression model. Furthermore, there were no acute DVT events and so the changes of inflammation parameters or SLE disease activity cannot be attributed to thrombosis occurrence. Moreover, we present a cross-sectional study and therefore the DVT events were retrospectively registered.

In conclusion, the $\operatorname{IgG}$ aPE titer was the best correlated with the history of DVT, so we might presume that the non-criteria IgG aPE might be involved in DVT pathogenesis in patients with SLE and APS. SLE disease activity proved to be also correlated with the history of DVT.

\section{Conflict of interest}

None to declare.

\section{Abbreviations}

Abs - antibodies

APLAs - antiphospholipid antibodies

APS - antiphospholipid syndrome

aPL - antiphospholipid

$\mathrm{aCL}$ - anticardiolipin

a $\beta 2$ GPI - anti- $\beta 2$ glycoprotein I

aPE - antiphosphatidylethanolamine

aPS - antiphosphatidylserine

aPS/PT - anti-prothrombin in complex with 
phosphatidylserine; aPT - antiprothrombin

APS - antiphospholipid syndrome

CRP - C-reactive protein

DVT - deep vein thrombosis

ESR - erythrocyte sedimentation rate

LAC - lupus anticoagulant

SLE - systemic lupus erythematosus

SLEDAI - Systemic Lupus Erythematosus Disease Activity Index

\section{References}

1. Miyakis S, Lockshin MD, Atsumi T, Branch DW, Brey RL, Cervera R, et al. International consensus statement on an update of the classification criteria for definite antiphospholipid syndrome (APS). J Thromb Haemost. 2006 Feb;4(2):295-306. DOI: 10.1111/j.15387836.2006.01753.x

2. Nayfe R, Uthman I, Aoun J, Saad Aldin E, Merashli M, Khamashta MA. Seronegative antiphospholipid syndrome. Rheumatology (Oxford). 2013 Aug;52(8):135867. DOI: $10.1093 /$ rheumatology/ket126

3. Staub HL, Bertolaccini ML, Khamashta MA. Anti-phosphatidylethanolamine antibody, thromboembolic events and the antiphospholipid syndrome. Autoimmun Rev. 2012 Dec;12(2):230-4. DOI: 10.1016/j. autrev.2012.07.008

4. Bertolaccini ML, Amengual O, Andreoli L, Atsumi T, Chighizola CB, Forastiero R, et al. 14th International Congress on Antiphospholipid Antibodies Task Force. Report on antiphospholipid syndrome laboratory diagnostics and trends. Autoimmun Rev. 2014 Sep;13(9):917-30. DOI: 10.1016/j.autrev.2014.05.001

5. Sestak A, O’Neil KM. Familial lupus and antiphospholipid syndrome. Lupus. 2007; 16(8): 556-63. DOI: 10.1177/0961203307078071

6. Bertolaccini ML, Gomez S, Pareja JF, Theodoridou A, Sanna G, Hughes GR, et al. Antiphospholipid antibody tests: spreading the net. Ann Rheum Dis. 2005 Nov;64(11):1639-43. DOI: 10.1136/ard.2005.035824

7. Szodoray P, Tarr T, Tumpek J, Kappelmayer J, Lakos $\mathrm{G}$, Poor G, et al. Identification of rare anti-phospholipid/protein co-factor autoantibodies in patients with systemic lupus erythematosus. Autoimmunity. 2009 Sep;42(6):497-506.DOI: 10.1080/08916930902882731

8. Petri M, Orbai AM, Alarcón GS, Gordon C, Merrill JT,
Fortin PR, et al. Derivation and validation of the Systemic Lupus International Collaborating Clinics classification criteria for systemic lupus erythematosus. Arthritis Rheum. 2012 Aug;64(8):2677-86. DOI: 10.1002/ art. 34473

9. Bombardier C, Gladman DD, Urowitz MB, Caron D, Chang CH. Derivation of the SLEDAI. A disease activity index for lupus patients. The Committee on Prognosis Studies in SLE. Arthritis Rheum. 1992 Jun;35(6):630-40. DOI: 10.1002/art.1780350606

10. Gavris C, Anghel M, Radoi M, Gheorghita E, Duca L, Pamfil G, et al. Risk of recurrent thrombosis related to antiphospholipid antibodies, soluble CD40L and P selectin serum levels in patients with antiphospholipid syndrome secondary to systemic lupus eritematosus. RRML. 2010 Sept;3(4):23-28.

11. Wahl DG, Guillemin F, de Maistre E, Perret C, Lecompte T, Thibaut G. Risk for venous thrombosis related to antiphospholipid antibodies in systemic lupus erythematosus-a meta-analysis. Lupus. 1997;6(5):46773. DOI: 10.1177/096120339700600510

12. Somers E, Magder LS, Petri M. Antiphospholipid antibodies and incidence of venous thrombosis in a cohort of patients with systemic lupus erythematosus. J Rheumatol. 2002 Dec;29(12):2531-6.

13. Petri M. Update on anti-phospholipid antibodies in SLE: the Hopkins' Lupus Cohort. Lupus. 2010 Apr;19(4):419-23. DOI: 10.1177/0961203309360541

14. Galli M, Luciani D, Bertolini G, Barbui T. Lupus anticoagulants are stronger risk factors for thrombosis than anticardiolipin antibodies in the antiphospholipid syndrome: a systematic review of the literature. Blood. 2003 Mar;101(5):1827-32. DOI: 10.1182/ blood-2002-02-0441

15. Bertolaccini ML. Antibodies to prothrombin. Lupus. 2012 Jun;21(7):729-31. DOI: 10.1177/0961203312443299

16. Bertolaccini ML, Murru V, Sciascia S, Sanna G, Khamashta MA. The clinical value of testing for antibodies to phosphatidylethanolamine (aPE) in patients with systemic lupus erythematosus (SLE). Thromb Res. 2012 Dec;130(6):914-8. DOI: 10.1016/j.thromres.2012.06.008

17. Sanmarco M, Gayet S, Alessi MC, Audrain M, de Maistre E, Gris JC, et al. Antiphosphatidylethanolamine antibodies are associated with an increased odds ratio for thrombosis. A multicenter study with the participation 
of the European Forum on antiphospholipid antibodies. Thromb Haemost. 2007 Jun;97(6):949-54. DOI: 10.1160/TH06-10-0604

18. Sanmarco M, Bardin N. The contribution of antiphosphatidylethanolamine antibodies in the diagnosis of the antiphospholipid syndrome. Lupus. 2012
Jun;21(7):727-8. DOI: 10.1177/0961203312437272

19. Matta BN, Uthman I, Taher AT, Khamashta MA. The current standing of diagnosis of antiphospholipid syndrome associated with systemic lupus erythematosus. Expert Rev Clin Immunol. 2013 Jul;9(7):659-68. DOI: 10.1586/1744666X.2013.811183 